https://doi.org/10.46344/JBINO.2021.v10i04.07

\title{
IN-SILICO DOCKING AND MOLECULAR DYNAMIC SIMULATION STUDIES OF NsP2 PROTEASE FROM CHIKUNGUNYA VIRUS
}

\author{
MahammadHussain Memon, Anjoomaara Patel, Riya Patel, *Sharav Desai and Dhananjay Meshram
}

Department of In-Silico Drug Discovery \& Design, Pioneer Pharmacy Degree College, Vadodara-390019, Gujarat, India.

Email: - sharavdesai@gmail.com

(Received on date: 17.05.2021

Date of Acceptance: 25.06.2021

Date of publication: 30.07 .2021$)$

\section{ABSTRACT}

Chikungunya virus (CHIKV) is an Arbovirus, transmitted to humans primarily by Aedes aegypti a species of mosquito. Infection due to this pathogen is often associated with fever, rash and arthralgia. The NsP2 protease of Chikunguniya virus has a crucial role in genome replication and hence acts as a promising drug target. We have used computer-aided drug design approach to find out the natural agents that can used in the treatment of Chikunguniya infection. We initially screened 162 natural compounds having antiviral activity. The 3D structures of these compounds were retrieved from PubChem Database. Molecular docking studies of these ligands were performed using PyRx ( $\vee$ 8.0) and, ADMET profiles were obtained by using SWISS ADME and data warrior tools. The obtained results after data analysis demonstrated that the ligand taxifolin has good binding affinity and complies with all the ADME parameters. The Molecular dynamic simulation studies of the taxifolin in complex with the PDB structure of NsP2 Protease of CHIKV (PDB ID: 3TRK) were carried out and the parameters like RMSD, RMSF, and radius of gyration were observed to understand the fluctuations and protein-ligand interaction.

Key Words: Molecular Docking, MD simulation, CHIKV, Protease, VMD and NAMD, RMSD 


\section{Introduction}

Chikungunya virus (CHIKV) is an arbovirus which belongs to the alphavirus genus, family Togaviridae. It has been responsible for major outbreak of devastating human arthritis. Chikunguniya fever caused by the virus was first described in 1952 during an epidemic in Newala district of Tanzania. "Chikunguniya" in Makonde language translates to "that which bends up" relating to the stopped posture (Her et al., 2009) developed as a result of rheumatologic inflammation.

CHIKV can be transmitted through an urban cycle, man to mosquito to man, or a sylvatic cycle, animal to mosquito to man (Chhabra et al., 2008). The virus is transmitted to humans by mosquitoes of the Aedes genus (Aedes furcifer in Africa and Aedes aegypti in Asia), similar to the dengue fever causing virus (Schwartz \& Albert, 2010).

The symptoms of "Chikunguniya fever" CHIKF infection generally start after 4-7 days of the mosquito bite. Infection presents in two phases, the first being acute, while the second stage is persistent (chronic), causing disabling polyarthritis (Ziegler et al., 2008). Acute infection lasts 1-10 days and is characterized by a painful polyarthralgia, high fever, asthenia (weakness), headache, vomiting, rash, and myalgia (muscle pain).

The CHIKV contains an RNA genome of approximately $11.8 \mathrm{kB}$ that is single stranded and messenger ('positive strand') sense. It consists of two open reading frames (ORFs) viz non-structural and structural. During viral replication, non-structural polyprotein proceeds to four non-structural protein (nsP1, nsP2, nsP3, nsP4) responsible for viral replication, RNA capping and invasion of the host defence mechanism. In addition the structural polyprotein proceeds' into various protein (C, E3, E2, 6K, El) that are involved in viral particle assembly (Weaver et al., 2012).

Currently, targeting viral enzymes that are crucial for viral replication is believed to be an attractive strategy for development of antiviral therapy. The nsP2 protein is a multifunctional protein. The proteolytic domain has been allocated to its C-terminal section which forms a papain like cysteine protease (also known as thiol protease).The nsP2 proteolytic activity is critical for virus replication and is responsible for cleavage of the non-structural polyprotein complex. The N-terminal having RNA-triphosphatase (RTPase) activity that perform the RNA-capping. It was also found to have the nucleotide triphosphatase (NTPase) activity. Both NTPase and RTPase activities are completely dependent on $\mathrm{Mg} 2+$ ions (Karpe et al., 2011). Therefore, the inhibition of NsP2 protein not only inhibits the NTPase and RTPase activity but also inhibits the virus replication.

So we have selected NsP2 protease enzyme as the drug target. The crystal structure PDB ID: 3TRK retrieved from the protein data bank was used here to find suitable ligands against it (Berman et al., 2000).

Currently, there is no specific antiviral drug and vaccine available in the market for Chikunguniya virus. Symptomatic treatment is available which include analgesic drug, NSAIDs and 
corticosteroids. As in vitro and in vivo screening of antiviral is laborious and time consuming, here we have performed insilico approach to find natural alternatives to treat the CHIKV infection. We initially selected natural compounds having antiviral activity, from literary sources and subjected them to molecular docking and molecular dynamic simulation studies to get to the lead natural candidate for treating Chikunguniya virus infection.

\section{Material and Methods \\ Protein structure preparation}

The X-ray diffraction-based crystal structure of the Chikunguniya Virus NsP2 Protease (PDB ID: 3TRK) in a complex with ligand $\mathrm{Na}^{+}$(sodium) with a resolution of $2.40 \AA$ was sourced from the protein data bank. The structure was cleaned to ensure maximum quality and reliability (Isa et al., 2019) by removing the bound ligands and water molecules. The missing atoms and residues were added to bridge any gaps in the protein molecule. Steric clashes were minimized by choosing proper orientation and polar hydrogen atoms were added to ascertain hydrogen atoms bonded to electronegative atoms like oxygen and nitrogen. Formal bond orders were determined, side chains were optimized and fixed, charges added using program implemented in chimera, SWISS PDB viewer, and Chiron minimization and refinement tool (Johansson et al., 2012, Porollo \& Meller, 2010, Ramachandran et al., 2011 ).

\section{Computational screening}

A thorough literature survey was conducted to find out the natural compounds having said antiviral properties. A total of 162 compounds were identified and the structures of the identified compounds were retrieved from the PubChem database. The compounds were imported in to the PyRx ( $V$ 8.0) and energy minimization was done using Open Babel (Version 2.3.1) (O'Boyle et al., 2011) module. Energy minimization was done via the Universal force field (UFF) using the conjugate gradient algorithm. A total number of 200 steps were set and the number of steps to update was set to 1. The minimization was set to stop at an energy difference of less than $0.1 \mathrm{Kcal} / \mathrm{mol}$ (Luo et al., 2020).

\section{Docking studies}

Molecular docking was performed with PyRx (V 8.0), which is an extension of the python molecular viewer. A Lamarckian genetic algorithm was used to perform the automated molecular docking of the protein with each ligand. The torsion bonds and side chains were kept to rotate freely, while the protein structure was kept rigid. Gasteiger charges were computed, and all the charges of nonpolar hydrogens were assigned (Morris et al., 1998). The grid map was set at $60 \times 60 \times 60 \times$ and the grid was spaced at $0.375 \AA$. Both selected ligand and protein were converted in to pdbat structure format. Protein and ligands were loaded in to PyRx as macromolecule and ligand, respectively. All the compounds were docked and affinity was calculated in kilocalories per mole (Gasteiger \& Marsili, 1980). 
Table 1. Molecular Properties \& Drug likeness of Selected Ligands.

\begin{tabular}{|c|c|c|c|c|c|c|}
\hline $\begin{array}{l}\text { Molecule } \\
\text { Name }\end{array}$ & PubChem ID & $\begin{array}{l}\text { Number of } \\
\text { HBA }\end{array}$ & $\begin{array}{l}\text { Number of } \\
\text { HBD }\end{array}$ & $\begin{array}{l}\text { Molecular } \\
\text { weight }\end{array}$ & $\operatorname{cLog} \mathrm{P}$ & $\begin{array}{l}\text { Drug } \\
\text { likeness }\end{array}$ \\
\hline 73659 & 73659 & 4 & 3 & 472.7 & 5.26 & -2.0276 \\
\hline 36462 & 36462 & 13 & 3 & 588.56 & 1.13 & -1.9839 \\
\hline 357293 & 357293 & 4 & 0 & 310.34 & 2.02 & -0.56695 \\
\hline 5271805 & 5271805 & 10 & 4 & 566.51 & 4.34 & 0.40331 \\
\hline 5281627 & 5281627 & 10 & 5 & 538.46 & 3.98 & 0.28194 \\
\hline 3663 & 3663 & 8 & 6 & 504.44 & 4.26 & -1.9057 \\
\hline 107876 & 107876 & 13 & 10 & 594.52 & 1.14 & 0.1505 \\
\hline 439501 & 439501 & 12 & 8 & 584.65 & -0.52 & 0.43549 \\
\hline 4978 & 4978 & 9 & 7 & 520.44 & 3.5 & -2.0279 \\
\hline 10097848 & 10097848 & & 6 & 510.49 & 3.9 & \\
\hline 10621 & 10621 & 15 & 8 & 610.56 & -1.06 & 2.0396 \\
\hline 5154 & 5154 & 4 & 0 & 332.33 & 2.88 & -2.4707 \\
\hline 5213 & 5213 & 10 & 5 & 482.44 & 1.59 & 0.22481 \\
\hline $1.3 \mathrm{E}+08$ & $1.3 \mathrm{E}+08$ & 19 & 12 & 651.48 & -1.13 & 0.62055 \\
\hline 222154 & 222154 & & 4 & 530.65 & 2.6 & 0.14199 \\
\hline 441688 & 441688 & 16 & 11 & 611.53 & -2.67 & -8.6993 \\
\hline 5280637 & 5280637 & 11 & 7 & 448.38 & 0.15 & -3.2535 \\
\hline 5282160 & 5282160 & 12 & 8 & 464.38 & -0.24 & -3.7091 \\
\hline 6473766 & 6473766 & 4 & 2 & 338.4 & 2.38 & -0.41 \\
\hline 46173996 & 46173996 & 11 & 9 & 562.52 & 2.34 & 0.43168 \\
\hline 471393 & 471393 & 10 & 7 & 442.37 & 1.24 & 0.37426 \\
\hline 5320826 & 5320826 & 13 & 9 & 480.38 & -0.72 & -3.7091 \\
\hline 64945 & 64945 & 3 & 2 & 456.7 & 5.93 & -3.658 \\
\hline 5280805 & 5280805 & 16 & 10 & 610.52 & -1.51 & 1.9337 \\
\hline 10494 & 10494 & 3 & 2 & 456.7 & 6.07 & -1.782 \\
\hline 5280343 & 5280343 & 7 & 5 & 302.24 & 1.23 & -0.082832 \\
\hline 5280804 & 5280804 & 12 & 8 & 464.38 & -0.48 & -3.6679 \\
\hline 5280863 & 5280863 & 6 & 4 & 286.24 & 1.58 & -0.082832 \\
\hline 5281670 & 5281670 & 7 & 5 & 302.24 & 1.2 & -0.082832 \\
\hline 5281718 & 5281718 & 8 & 6 & 390.38 & 0.64 & -4.3466 \\
\hline 5318585 & 5318585 & 6 & 4 & 354.35 & 3.09 & -0.33653 \\
\hline
\end{tabular}

2021 July Edition |www.jbino.com | Innovative Association 


\begin{tabular}{|c|c|c|c|c|c|c|}
\hline 10168 & 10168 & 6 & 3 & 284.22 & 1.48 & -1.1733 \\
\hline 16203170 & 16203170 & 12 & 11 & 580.54 & 1.68 & 0.31525 \\
\hline 439533 & 439533 & 7 & 5 & 304.25 & 0.51 & 0.44477 \\
\hline 5280794 & 5280794 & 1 & 1 & 412.69 & 6.98 & 1.2217 \\
\hline 5281614 & 5281614 & 6 & 4 & 286.24 & 1.55 & -0.082832 \\
\hline 5281616 & 5281616 & 5 & 3 & 270.24 & 1.99 & -0.082832 \\
\hline 246330 & 246330 & 6 & 4 & 288.25 & 0.91 & 0.44477 \\
\hline 275196 & 275196 & 8 & 0 & 413.42 & 2.61 & 4.3358 \\
\hline 440832 & 440832 & 5 & 4 & 271.24 & 0.73 & -6.14 \\
\hline 442428 & 442428 & 14 & 8 & 580.53 & -0.87 & 0.64246 \\
\hline 5317435 & 5317435 & 6 & 4 & 288.25 & 0.89 & 0.44477 \\
\hline 10607 & 10607 & 8 & 1 & 414.41 & 2.33 & 0.17227 \\
\hline 345501 & 345501 & 7 & 0 & 398.41 & 3.08 & 0.17601 \\
\hline 3503 & 3503 & 8 & 6 & 518.55 & 5.04 & -4.3442 \\
\hline 444170 & 444170 & 18 & 8 & 663.43 & -3.77 & -29.561 \\
\hline 5281612 & 5281612 & 6 & 3 & 300.26 & 2.19 & 0.40331 \\
\hline 5281665 & 5281665 & 6 & 4 & 286.24 & 1.72 & 0.28194 \\
\hline 5281672 & 5281672 & 8 & 6 & 318.24 & 0.79 & -0.082832 \\
\hline 9890209 & 9890209 & 3 & 1 & 454.68 & 5.95 & -5.7793 \\
\hline 11385155 & 11385155 & 5 & 2 & 440.57 & 4.36 & -1.4045 \\
\hline 11729855 & 11729855 & 5 & 1 & 438.56 & 4.54 & -2.7591 \\
\hline 12315393 & 12315393 & 4 & 2 & 384.51 & 3.68 & -0.95205 \\
\hline 12315515 & 12315515 & 4 & 2 & 474.72 & 5.94 & -20.119 \\
\hline 16760705 & 16760705 & 6 & 2 & 470.6 & 3.42 & 1.6889 \\
\hline 5280445 & 5280445 & 6 & 4 & 286.24 & 1.73 & 0.28194 \\
\hline 5281697 & 5281697 & 6 & 4 & 286.24 & 1.81 & 0.28194 \\
\hline 72281 & 72281 & 6 & 3 & 302.28 & 1.91 & -0.0783 \\
\hline 72435 & 72435 & 8 & 1 & 414.41 & 2.32 & 0.17227 \\
\hline $1.02 \mathrm{E}+08$ & $1.02 \mathrm{E}+08$ & 3 & 3 & 460.73 & 5.77 & -2.0603 \\
\hline 10219 & 10219 & 6 & 1 & 480.64 & 4.24 & 3.8313 \\
\hline 12315516 & 12315516 & 4 & 2 & 474.72 & 5.98 & -20.119 \\
\hline 5280459 & 5280459 & 11 & 7 & 448.38 & 0.22 & 1.9289 \\
\hline 5281855 & 5281855 & 8 & 4 & 302.19 & 1 & -1.5983 \\
\hline 932 & 932 & 5 & 3 & 272.25 & 1.84 & -0.22006 \\
\hline 21582894 & 21582894 & 2 & 1 & 442.72 & 6.5 & -4.2965 \\
\hline 5281607 & 5281607 & 4 & 2 & 254.24 & 2.55 & 0.28194 \\
\hline 5281643 & 5281643 & 12 & 8 & 464.38 & -0.38 & -3.6679 \\
\hline 5281647 & 5281647 & 11 & 8 & 422.34 & -0.77 & -3.0467 \\
\hline 5282102 & 5282102 & 11 & 7 & 448.38 & -0.09 & -3.6679 \\
\hline 92765 & 92765 & 4 & 0 & 377.48 & 4.34 & 0.52242 \\
\hline 24360 & 24360 & 5 & 1 & 348.35 & 2.2 & 5.3292 \\
\hline 3220 & 3220 & 5 & 3 & 270.24 & 1.87 & -1.1275 \\
\hline 443013 & 443013 & 8 & 1 & 414.41 & 2.32 & 0.17227 \\
\hline
\end{tabular}

2021 July Edition |www.jbino.com | Innovative Association 


\begin{tabular}{r|r|}
13893946 & 13893946 \\
215159 & 215159 \\
40305 & 40305 \\
442835 & 442835 \\
5280961 & 5280961 \\
72276 & 72276 \\
259846 & 259846 \\
3884 & 3884 \\
44584517 & 44584517 \\
11012233 & 11012233 \\
5281677 & 5281677 \\
5487772 & 5487772
\end{tabular}

\begin{tabular}{l|l|}
1 & 1 \\
7 & 0 \\
4 & 1 \\
7 & 1 \\
5 & 0 \\
6 & 3 \\
1 & 5 \\
3 & 1 \\
2 & 1 \\
2 & 1 \\
7 & 2 \\
5 & 2 \\
& 2
\end{tabular}

426.72
369.37
350.39
400.42
270.24
290.27
426.72
242.27
442.72
306.48
344.32
301.29

7.51
2.89
2.69
3.3
2.04
0.85
7.28
2.54
6.81
3.88
2.61
2.04

$-5.3696$

2.7556

$-2.1697$

0.34682

$-0.093853$

0.31525

$-22.172$

$-3.8914$

$-2.8027$

$-0.37188$

$-0.10513$

1.1795

\section{ADME and Toxicity predictions}

The compounds with good binding energies were further studied for their adsorption, distribution, excretion, metabolism, and toxicity profile using SWISS ADME(Daina et al., 2017) and data warrior tools (Sander et al.,2009, Sander et al., 2015). The predicted properties considered were blood-brain barrier penetration properties, human intestinal

absorption, inhibition to cytochrome P450 enzyme and PGP substrate binding. Compounds showing satisfactory properties were further studied for their toxicity profile using data warrior tools. Toxicity profiles included were mutagenicity, tumorigenicity, irritability, reproducibility, Ames toxicity and carcinogenesis.

Table 2. ADMET analysis with the Lowest Binding affinity.

\begin{tabular}{r|l|l|l|l|l|l|l|l|l} 
PubChem ID & $\begin{array}{l}\text { BBB } \\
\text { Penetration }\end{array}$ & HIA & $\begin{array}{l}\text { CYP 2D6 } \\
\text { Inhibitor }\end{array}$ & $\begin{array}{l}\text { PGP } \\
\text { substrate } \\
\text { binding }\end{array}$ & $\begin{array}{l}\text { Mutageni } \\
\text { c }\end{array}$ & Tumorigenic & $\begin{array}{l}\text { Reprodu } \\
\text { ctive } \\
\text { effect }\end{array}$ & Irritant & $\begin{array}{l}\text { Binding } \\
\text { affinity }\end{array}$ \\
73659 & No & High & No & Yes & none & None & none & None & -13.2 \\
36462 & No & Low & Yes & Yes & none & None & none & None & -12.3 \\
357293 & Yes & High & Yes & No & none & None & none & High & -10 \\
5271805 & No & Low & No & No & none & None & none & None & -9.6 \\
5281627 & No & Low & No & No & none & None & high & None & -9.4 \\
3663 & No & Low & No & No & low & High & none & High & -9.3 \\
107876 & No & Low & No & No & none & None & none & None & -8.8 \\
439501 & No & Low & No & No & none & None & none & None & -8.8 \\
4978 & No & Low & No & No & high & High & none & High & -8.8 \\
10097848 & No & Low & No & No & none & None & none & None & -8.7 \\
10621 & No & Low & No & Yes & none & None & none & None & -8.7 \\
5154 & Yes & High & No & Yes & none & None & none & None & -8.7 \\
5213 & No & Low & No & No & none & None & none & None & -8.6 \\
129693153 & No & Low & No & Yes & none & None & none & None & -8.5 \\
222154 & No & High & Yes & Yes & none & None & high & None & -8.5
\end{tabular}




\begin{tabular}{|c|c|c|c|c|c|c|c|c|c|}
\hline 441688 & No & Low & No & No & none & None & none & None & -8.5 \\
\hline 5280637 & No & Low & No & Yes & none & None & none & None & -8.5 \\
\hline 5282160 & No & Low & No & Yes & high & None & none & None & -8.5 \\
\hline 6473766 & Yes & High & Yes & Yes & none & None & none & None & -8.5 \\
\hline 46173996 & No & Low & No & No & none & None & none & None & -8.4 \\
\hline 471393 & No & Low & No & No & none & None & none & None & -8.4 \\
\hline 5320826 & No & Low & No & No & high & None & none & None & -8.2 \\
\hline 64945 & No & Low & No & No & none & None & none & None & -8.2 \\
\hline 5280805 & No & Low & No & Yes & none & None & none & None & -8.1 \\
\hline 10494 & No & Low & No & No & none & None & none & None & -8 \\
\hline 5280343 & No & High & Yes & No & high & High & none & None & -8 \\
\hline 5280804 & No & Low & No & No & none & None & none & None & -8 \\
\hline 5280863 & No & High & Yes & No & high & None & none & None & -8 \\
\hline 5281670 & No & High & Yes & No & high & None & none & None & -8 \\
\hline 5281718 & No & High & No & Yes & none & None & high & None & -8 \\
\hline 5318585 & No & High & Yes & No & high & None & none & None & -8 \\
\hline 10168 & No & High & No & No & none & None & none & High & -7.9 \\
\hline 16203170 & No & Low & No & No & none & None & none & None & -7.9 \\
\hline 439533 & No & High & No & No & none & None & none & None & -7.9 \\
\hline 5280794 & No & Low & No & No & none & None & none & None & -7.9 \\
\hline 5281614 & No & High & Yes & No & high & None & none & None & -7.9 \\
\hline 5281616 & No & High & Yes & No & high & None & none & None & - \\
\hline 246330 & No & High & No & No & none & None & none & None & -7.8 \\
\hline 275196 & No & High & Yes & No & none & None & none & None & -7.8 \\
\hline 440832 & No & High & No & Yes & none & None & none & None & -7.8 \\
\hline 442428 & No & Low & No & Yes & none & None & none & None & -7.8 \\
\hline 5317435 & No & High & No & No & none & None & none & None & -7.8 \\
\hline 10607 & No & High & Yes & No & none & None & high & None & -7.7 \\
\hline 345501 & Yes & High & Yes & No & none & None & high & None & -7.7 \\
\hline 3503 & No & Low & No & No & low & High & none & None & -7.7 \\
\hline 444170 & No & Low & No & No & none & None & none & None & -7.7 \\
\hline 5281612 & No & High & Yes & No & none & None & none & None & -7.7 \\
\hline 5281665 & No & High & Yes & No & high & None & none & None & -7.7 \\
\hline 5281672 & No & Low & No & No & high & None & none & None & -7.7 \\
\hline 9890209 & No & Low & No & No & none & None & none & None & -7.7 \\
\hline 11385155 & No & High & No & Yes & none & None & none & None & -7.6 \\
\hline 11729855 & No & High & No & Yes & none & None & none & None & -7.6 \\
\hline 12315393 & Yes & High & No & Yes & none & None & high & None & -7.6 \\
\hline 12315515 & No & Low & No & No & none & None & none & None & -7.6 \\
\hline 16760705 & No & High & No & Yes & none & None & low & None & -7.6 \\
\hline 5280445 & No & High & Yes & No & none & None & none & None & -7.6 \\
\hline 5281697 & No & High & Yes & No & none & None & none & None & -7.6 \\
\hline 72281 & No & High & No & Yes & none & None & none & None & -7.6 \\
\hline
\end{tabular}




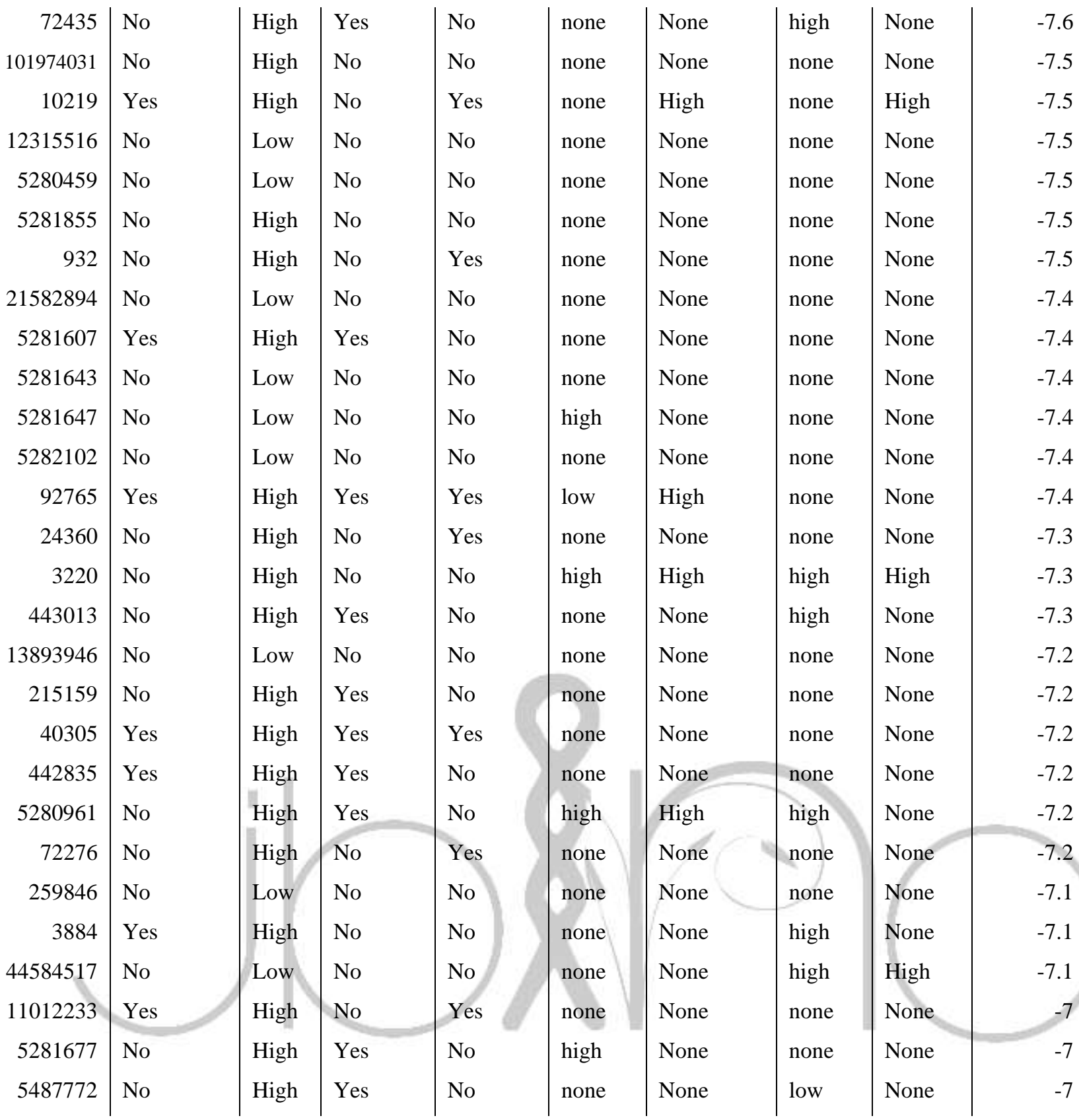

\section{Molecular dynamic simulation}

Docked protein and ligand complex was subjected to molecular dynamics simulation using NAMD software (Phillips et al., 2020). The success of MD simulation depends on the selection of the initial protein and ligand structures. Initially, the structure was checked for inconsistencies. Out of 86 compounds selected from the docking results, we have selected the Taxifolin with PubChem number 439533 as the best ligand. The docked complex was studied for its stability during the simulation. The root means square

deviation, root mean square fluctuation, and radius of gyration was studied for protein backbone residue and ligand within the binding site of the simulated system (Bornot et al., 2011, Kufareva \& Abagyan, 2012, Lobanov et al., 2008).The stability of the complex was examined by monitoring its root mean square deviation (RMSD) during 50,00,000 steps for a $10 \mathrm{~ns}$ simulation. MD simulations were performed using the CHARMM36 force field (Soteras et al., 2016). Visual molecular dynamics (VMD) was used to generate PSF files for the complex 
(Sander et al., 2009). The complex was solvated in cubic water boxes containing transferable intermolecular potential with 3 points (TIP3P) water molecules. The box size was chosen to match the molecular dimensions so that there was a distance of $5^{\circ} \mathrm{A}$ between the protein surface and the edges of the periodic box. A $5^{\circ} \mathrm{A}$ cut off distance was used to calculate shortrange non-bonded interactions. The particle mesh Ewald (PME) method was used to calculate long-range electrostatic interactions. The SHAKE method was used to constrain all the bonds involving hydrogen atoms. A conjugated gradient system was used for energy minimization, with all parameters set to default. The system first performed 10000 steps of Conjugated gradient with energy minimization. We used Langevin Dynamics with pressure control so our system was not an NVT ensemble. The Nose-Hoover method was used to maintain a constant temperature. The time step of each simulation was set to 2 fs (Phillips et al., 2020, Phillips et al., 2005, Ribeiro et al., 2016). Visualizations and data analysis were performed with VMD software (Hsin et al., 2008).

\section{Results and discussion}

\section{Virtual screening and docking results}

Virtual screening helps us to screen the biological molecules with good binding affinity. In this study, we have used PyRx 8.0 tool to screen out the molecules. A total of 162 natural ligands were selected and were docked to the target protein.

The docked compounds were examined in the Auto dock tool and binding free energy was calculated (Cosconati et al., 2010, Morris et al., 2009). We have selected a total of 86 compounds on their binding affinity ranging from -13.2 to $-7.0 \mathrm{kcal} / \mathrm{mol}$ (Table 2).

\section{ADMET analysis}

We had selected 86 compounds and the same compounds were studied for their ADMET properties. The properties like human intestinal absorption, irritability, reproductive effect, inhibition to cytochrome p450 enzyme, and several others were predicted. It was clear from the results that compound number 439533, 246330, 275196, 5317435 and 101974031 have a high intestinal absorption value. From the Table 2, it was also observed that we had several compounds like 357293, 222154, 73659, 11385155, and 11729855 have high intestinal absorption values but at the same time they are also showing their inhibitory properties towards the Cytochrome P450 enzymes and PGP substrate binding, so such compounds were removed from the further selections. Similarly, from the selected compounds with high intestinal absorption values and negative inhibitory actions to cytochrome P450 enzymes, compounds were also studied for their mutagenic, tumorigenic, irritability, and reproductive effect. So the compounds having either of the side effects were also removed from the study. Finally, we selected four ligands namely Taxifolin_CID_439533, Dihydrofisetin_CID_246330,

Narcotine_CID_275196

and

Fustin_CID_5317435 which complied all the parameters of ADMET. Among these we selected Taxifolin_CID_439533 for further analysis considering its higher binding affinity compared to other three compounds. The ligand selected for further study was having hydrogen bonds and also presents a hydrophobic 
interaction with the protein Table 3 \&Table 4.

\section{Protein-ligand interaction}

The hydrogen bond and hydrophobic interactions of protein-ligand complexes were analyzed by LigPlot+ ( $v$ 1.4.5)(38) and Protein-ligand interaction profiler. "LigPlott" is a graphical system that generates multiple three-dimensional (3D) diagram of ligand-protein interactions from docked complexes. PLIP is complementary to another state of the art tools like a Swiss dock, galaxy site, or ProBis and thus it can be used to study the protein-ligand complex. The server allows comprehensive detection and visualization of protein and ligand complexes along with interaction patterns.

Table 3. Hydrophobic Interaction between Protein and Ligand complex.

\begin{tabular}{|l|l|l|l|l|l|}
\hline Ligand & Residue & AA & Distance & Ligand Atom & Protein Atom \\
\hline \multirow{5}{*}{ Taxifolin } & $1191 \mathrm{~A}$ & PRO & 3.46 & 3216 & 1873 \\
& $1191 \mathrm{~A}$ & PRO & 3.84 & 3201 & 1874 \\
& $1191 \mathrm{~A}$ & PRO & 3.58 & 3215 & 1874 \\
& $1203 \mathrm{~A}$ & LEU & 3.58 & 3199 & 1988 \\
& $1203 \mathrm{~A}$ & LEU & 3.62 & 3212 & 1988 \\
& $1221 \mathrm{~A}$ & ILE & 3.54 & 3209 & 2154 \\
& $1239 \mathrm{~A}$ & LYS & 3.43 & 3219 & 2344 \\
& $1243 \mathrm{~A}$ & LEU & 3.67 & 3219 & 2388 \\
\hline
\end{tabular}

Table 4. Hydrogen bond interaction between Ligand and Protein complex.

\begin{tabular}{|c|c|c|c|c|c|c|c|c|c|}
\hline $\begin{array}{l}\text { Ligand } \\
\text { Taxifolir }\end{array}$ & $\begin{array}{l}\text { Residue } \\
\text { 1203A }\end{array}$ & $\begin{array}{l}\text { AA } \\
\text { LEU }\end{array}$ & $\begin{array}{l}\text { Distance } \\
\text { H-A }\end{array}$ & $\begin{array}{l}\text { Distanc } \\
\text { D-A }\end{array}$ & $\begin{array}{l}\text { Donor } \\
\text { Angle } \\
115.54\end{array}$ & $\begin{array}{l}\text { Protein } \\
\text { donor } \\
x\end{array}$ & $\begin{array}{l}\text { Side } \\
\text { chai } \\
x\end{array}$ & $\begin{array}{l}\text { Donor } \\
\text { Atom } \\
3193[\mathrm{O} 3\end{array}$ & $\begin{array}{l}\text { Acceptor } \\
\text { Atom } \\
1987[O 2]\end{array}$ \\
\hline
\end{tabular}




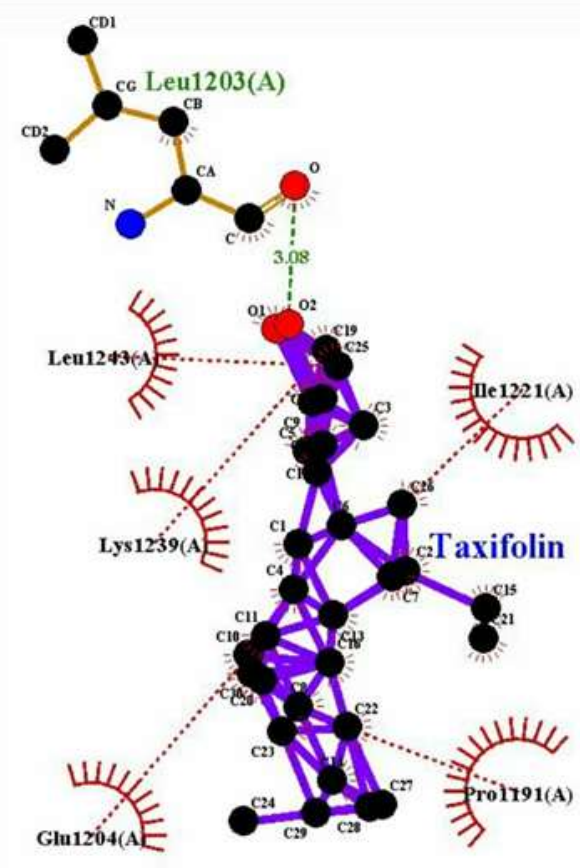

\section{3ttk_Docked}

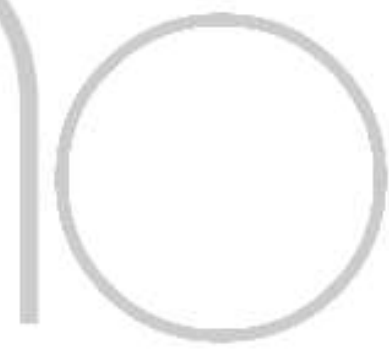

Figure 1. Protein and Ligand interaction Diagram.

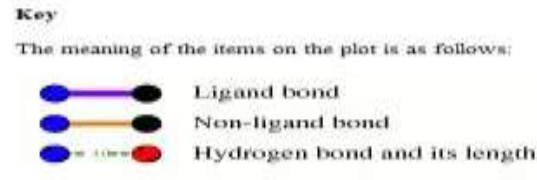

\section{Molecular Dynamic Simulation studies}

We assessed the residue RMSD to study the residue behaviour of the protein during the simulations. In general, a residue's RMSD value was considered to represent the local flexibility of a protein and ligand complex. It reflected the mobility of an atom during the MD
Non-1igand residuex involved in hydrophobic

Corresponding atoms involved in hydrophobie contact(s)

simulation trajectory. Therefore, a higher residue RMSD value indicated higher mobility; conversely, a lower residue RMSD value indicates lower mobility. To investigate the fluctuations in the ligandbinding energy as well as the motions of the amino acid residues within the complex during the simulation, the root 
means square fluctuation (RMSF) of the complex was also monitored. Besides, the compactness of the complex was determined by carefully examining how folded or unfolded the protein-ligand complex was by calculating the radius of gyration (Lobanov et al., 2008). Based on the docking analysis 86 compounds were selected for further ADMET investigation and it leads us to select the final compound (Taxifolin_CID_439533) to consider the structural stability of the protein-ligand complex by molecular dynamic simulation. The stability of the complex (3TRK_Taxifolin) was monitored using root mean square deviation (RMSD) during 10 ns simulation studies.

Table 5. RMSD values for the simulated complexes.

\begin{tabular}{l|l|l|l}
\hline Protein-ligand complex & Mean RMSD $(\AA)$ & Min RMSD $(\AA)$ & $\operatorname{Max} \operatorname{RMSD}(\AA)$ \\
\hline 3TRK_Taxifolin & 2.018 & 0.057 & 3.074
\end{tabular}

The values presented in (Table 5) for the protein-ligand complex studied for its stability during $10 \mathrm{~ns}$ simulation. From the values, it is clear that the range of RMSD obtained for the complex complies with the acceptance range of 1 to $3.5(\AA)$. It is also observed from the graphs that the complex was also equilibrated as the average RMSD values are stabilized at the end of the 10 ns simulation. This fixed range of RMSD was indicating the interaction between bound ligand and flexible loop region, as it reduces the flexibility of the protein-ligand complex. 


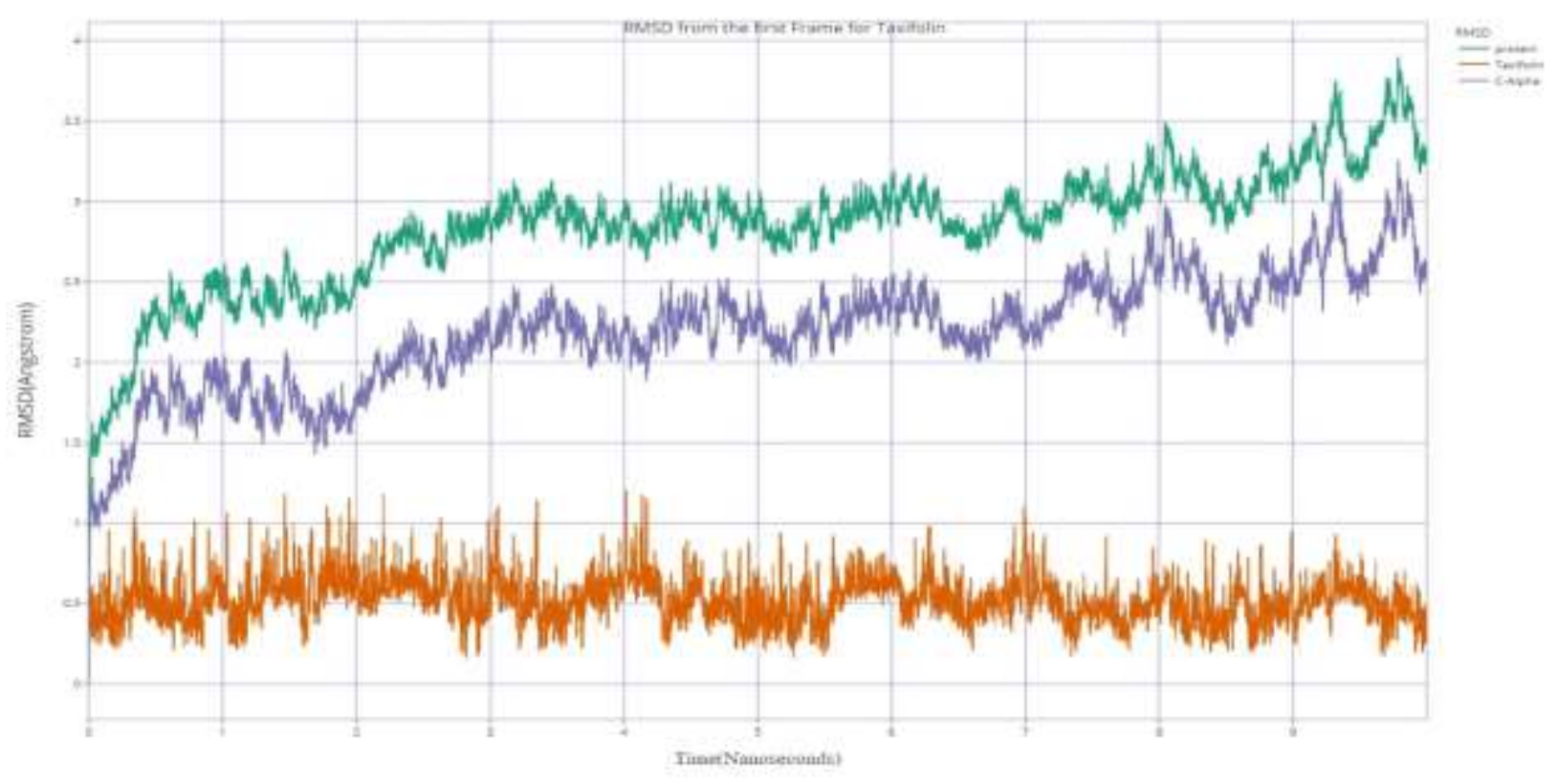

Figure 2. RMSD results for Taxifolin with 3 TRK protein, based on 10 ns simulation.

The root means square fluctuations (RMSF) were assessed and plotted to equate the flexibility of the residue in the ligand-protein complexes. The RMSF of the protein-ligand complex denoted the minimized fluctuation for the complex. The RMSF did not deviate much during the simulation period of $10 \mathrm{~ns}$ and the average RMSF values were kept constant for the complex.
The radius of gyration was also monitored during the 10-nsMD simulation for the protein-ligand complex to ascertain whether the complex was stably folded or unfolded. If the radius of gyration remained relatively constant, the complex was considered to be stably folded, otherwise, it was considered to be unfolded. 


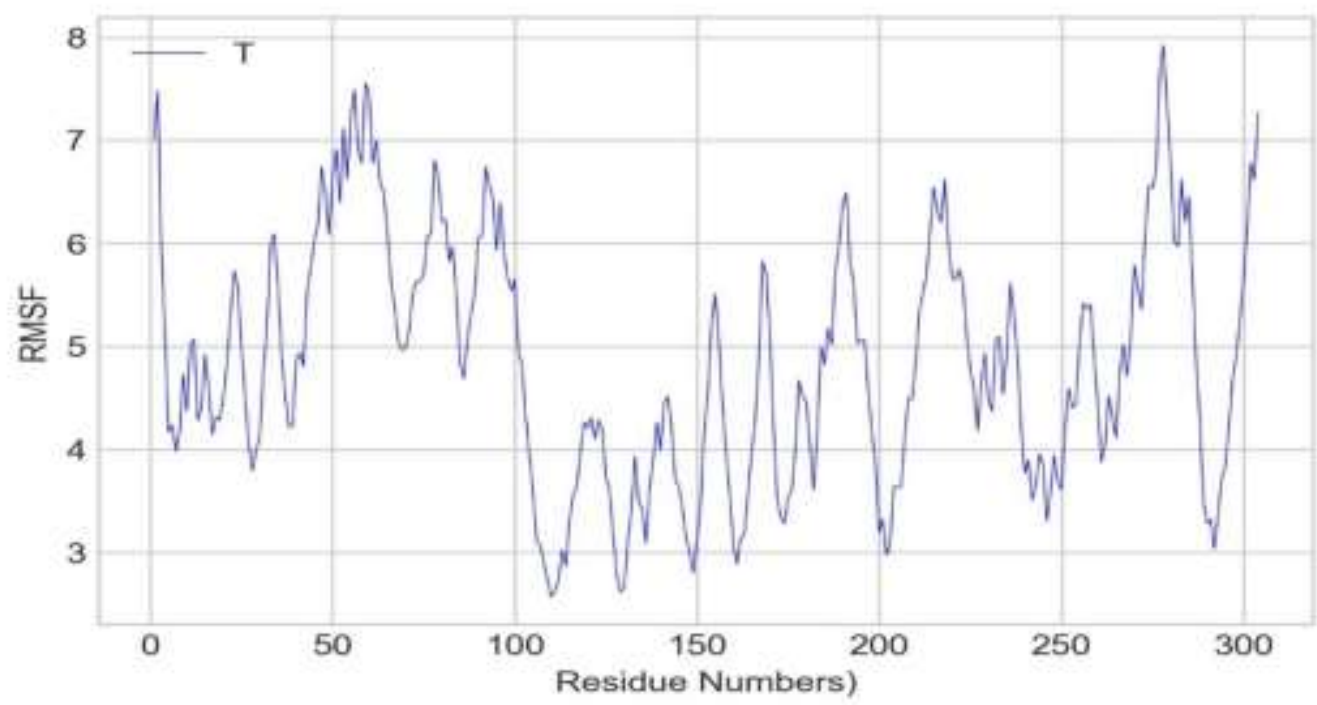

Figure 3. RMSF results for Taxifolin with 3TRK protein, based on the data from $10 \mathrm{~ns}$ simulation.

Table 6. The radius of Gyration for Protein-Ligand complexes.

\begin{tabular}{l|l|l|l}
\hline Protein-Ligand complex & Mean & Min & Max \\
\hline 3TRK_Taxifolin & 21.257 & 20.343 & 21.963
\end{tabular}

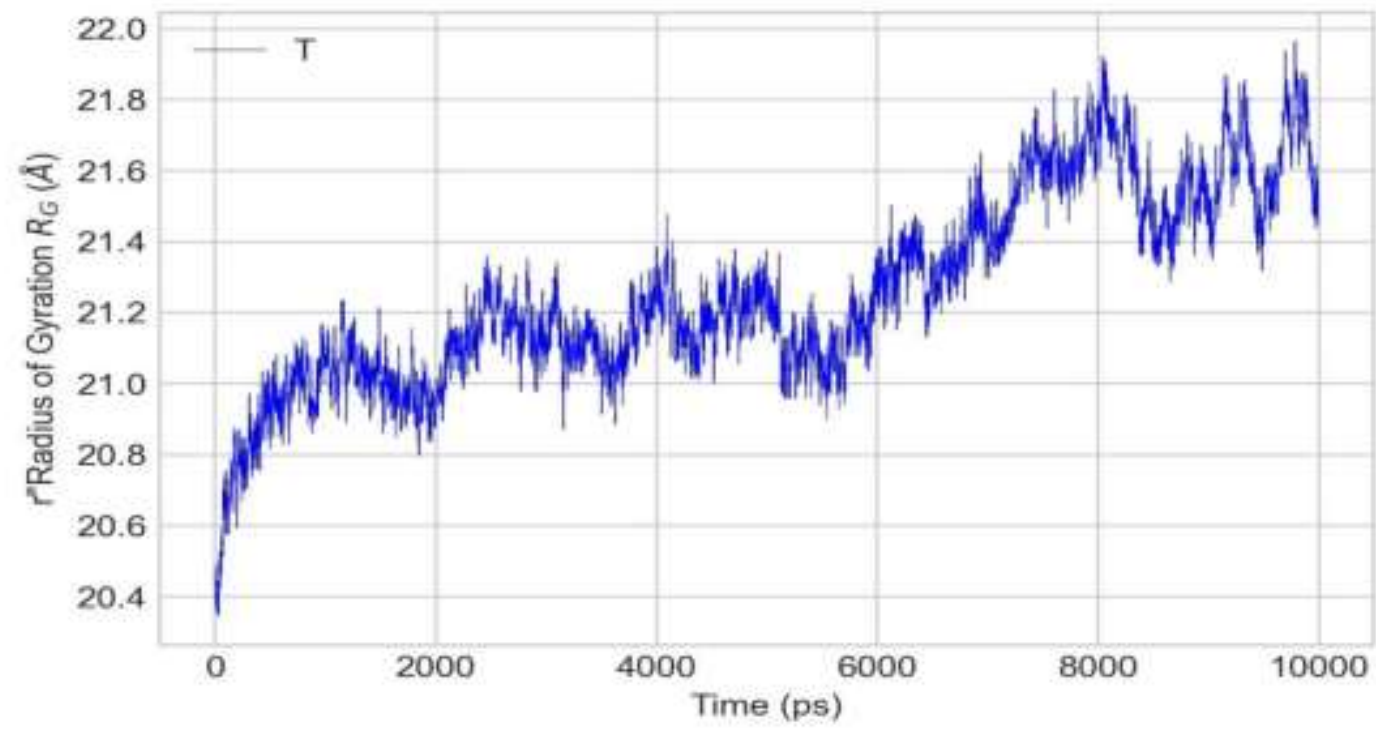

Figure 4. The radius of Gyration results for Taxifolin with 3TRK protein, based on the data from the 10 ns simulation.

In this study, the radius of gyration values obtained is listed in Table 6. All the values obtained for the test ligand Taxifolin showed a relatively constant radius gyration during the simulation. So, we can conclude that all of the complexes formed relatively stable folded 
polypeptide structures during the 10-ns MD simulation.

\section{CONCLUSION}

In our entire study, we tried to come up with an active natural compound for the treatment of Chikunguniya infection. We investigated 162 natural compounds for their activity against NsP2 Protease of the Chikunguniya Virus through computational methods including molecular docking and ADMET profiling using available software, and found four compounds (Taxifolin_CID_439533, Dihydrofisetin_CID_246330,

Narcotine_CID_275196

and

Fustin_CID_5317435) that complied with all the parameters. Among these we selected Taxifolin_CID_439533 for further analysis considering its higher binding affinity compared to Dihydrofisetin_CID_246330,

Narcotine_CID_275196

and fustin_CID_531735. Further the molecular dynamic simulation of 3TRK_Taxifolin complex was carried out to study its structural stability by investigating its RMSD, RMSF and radius of gyration values. By these investigations we conclude that Taxifolin can be a potential drug against Chikunguniya Virus (CHIKV).

\section{REFERENCES}

- Berman, H. M., Westbrook, J., Feng, Z., Gilliland, G., Bhat, T. N., Weissig, H., Shindyalov, I. N., \& Bourne, P. E. (2000). The Protein Data Bank. Nucleic acids research, 28(1),

235-242.

https://doi.org/10.1093/nar/28.1.235

- Bornot, A., Etchebest, C., \& de Brevern, A. G. (2011). Predicting protein flexibility through the prediction of local structures. Proteins, 79(3),

839-852. https://doi.org/10.1002/prot.22922

- Chhabra, M., Mittal, V., Bhattacharya, D., Rana, U., \& Lal, S. (2008). Chikungunya fever: a re-emerging viral infection. Indian journal of medical microbiology, 26(1), 512. https://doi.org/10.4103/02550857.38850

- Cosconati, S., Forli, S., Perryman, A. L., Harris, R., Goodsell, D. S., \& Olson, A. J. (2010). Virtual Screening with AutoDock: Theory and Practice. Expert opinion on drug discovery, 5(6), 597-607. https://doi.org/10.1517/17460441.2010.484 460

- Daina, A., Michielin, O., \& Zoete, V. (2017). SwissADME: a free web tool to evaluate pharmacokinetics, drug-likeness and medicinal chemistry friendliness of small molecules. Scientific Reports, 7(1). doi:10.1038/srep42717

Gasteiger, J., \& Marsili, M. (1980). Iterative partial equalization of orbital electronegativity-a rapid access to atomic charges. Tetrahedron, 36(22), 3219-3228. doi:10.1016/0040-

4020(80)80168-2

- Her, Z., Kam, Y. W., Lin, R. T., \& Ng, L. F. (2009). Chikungunya: a bending reality. Microbes and infection, 11 (14-15), 1165-1176.

https://doi.org/10.1016/j.micinf.2009.09.00 4

- Hsin, J., Arkhipov, A., Yin, Y., Stone, J. E., \& Schulten, K. (2008). Using VMD: an introductory tutorial. Current protocols in bioinformatics, Chapter 5, Unit-5.7. https://doi.org/10.1002/0471250953.bi050 $7 s 24$

- Isa, M. A., Majumdar, R. S., \& Haider, S. (2019). In silico identification of potential inhibitors against shikimate dehydrogenase through virtual screening and toxicity studies for the treatment of am | Innovative Association 
tuberculosis. International microbiology : the official journal of the Spanish Society for Microbiology, 22(1),

$7-17$. https://doi.org/10.1007/s10123-018-0021-2

- Johansson, M. U., Zoete, V., Michielin, O., \& Guex, N. (2012). Defining and searching for structural motifs using DeepView/SwissPdbViewer. BMC Bioinformatics, 13(1), 173. doi:10.1186/1471-2105-13-173

- Karpe, Y. A., Aher, P. P., \& Lole, K. S. (2011). NTPase and 5'-RNA Triphosphatase Activities of Chikungunya Virus nsP2 Protein. PLOS ONE, 6(7), e22336. doi:10.1371/journal.pone.0022336

- Kufareva, I., \& Abagyan, R. (2012). Methods of protein structure comparison. Methods in molecular biology (Clifton, N.J.), 857, 231-257. https://doi.org/10.1007/978-1-61779-5886_10

- Lobanov, M., Bogatyreva, N. S., \& Galzitskaia, O. V. (2008). Molekuliarnaia biologiia, 42(4), 701-706.

- Luo, S., Lenon, G. B., Gill, H., Hung, A., Dias, D. A., Li, M., \& Nguyen, L. T. (2020). Inhibitory effect of a weight-loss Chinese herbal formula RCM-107 on pancreatic a-amylase activity: Enzymatic and in silico approaches. PLOS ONE, 15(4),

e0231815. doi:10.1371/journal.pone.02318 15

- Morris, G. M., Goodsell, D. S., Halliday, R. S., Huey, R., Hart, W. E., Belew, R. K., \& Olson, A. J. (1998). Automated docking using a Lamarckian genetic algorithm and an empirical binding free energy function. Journal of Computational Chemistry, 19(14), 16391662. doi:10.1002/(sici) 1096-

987x(19981 115) 19:14<1639::aidjcc10>3.0.co;2-b

- Morris, G. M., Huey, R., Lindstrom, W., Sanner, M. F., Belew, R. K., Goodsell, D. S.,
\& Olson, A. J. (2009). AutoDock4 and AutoDockTools4: Automated docking with selective receptor flexibility. Journal of computational chemistry, 30(16), 27852791. https://doi.org/10.1002/jcc.21256

- O'Boyle, N. M., Banck, M., James, C. A., Morley, C., Vandermeersch, T., \& Hutchison, G. R. (2011). Open Babel: An open chemical toolbox. Journal of Cheminformatics,

33. doi:10.1 186/1758-2946-3-33 $3(1)$,

- Phillips, J. C., Braun, R., Wang, W., Gumbart, J., Tajkhorshid, E., Villa, E., Chipot, C., Skeel, R. D., Kalé, L., \& Schulten, K. (2005). Scalable molecular dynamics with NAMD. Journal of computational chemistry, 26(16), 17811802. https://doi.org/10.1002/jcc.20289

Phillips, J. C., Hardy, D. J., Maia, J. D. C., Stone, J. E., Ribeiro, J. V., Bernardi, R. C., ... Tajkhorshid, E. (2020). Scalable molecular dynamics on CPU and GPU architectures with NAMD. The Journal of Chemical Physics,

153(4), 044130. doi:10.1063/5.0014475.

Porollo, A., \& Meller, J. (2010). POLYVIEWMM: web-based platform for animation and analysis of molecular simulations. Nucleic acids research, 38(Web Server issue), W662W666. https://doi.org/10.1093/nar/gkq445

- Ramachandran, S., Kota, P., Ding, F., \& Dokholyan, N. V. (2011). Automated minimization of steric clashes in protein structures. Proteins, 79(1), 261-270. https://doi.org/10.1002/prot.22879

- Ribeiro, J. V., Bernardi, R. C., Rudack, T., Stone, J. E., Phillips, J. C., Freddolino, P. L., \& Schulten, K. (2016). QwikMD Integrative Molecular Dynamics Toolkit for Novices and Experts. Scientific Reports, 6(1). doi:10.1038/srep26536

- Sander, T., Freyss, J., von Korff, M., \& Rufener, C. (2015). DataWarrior: an open- 
source program for chemistry aware data visualization and analysis. Journal of chemical information and modeling, 55(2), 460-473. https://doi.org/10.1021/ci500588j

- Sander, T., Freyss, J., von Korff, M., Reich, J. R., \& Rufener, C. (2009). OSIRIS, an entirely in-house developed drug discovery informatics system. Journal of chemical information and modeling, 49(2), 232-246. https://doi.org/10.1021/ci800305f

- Schwartz, O., \& Albert, M. L. (2010). Biology and pathogenesis of chikungunya virus. Nature reviews. Microbiology, 8(7), 491-500. https://doi.org/10.1038/nrmicro2368

- Soteras Gutiérrez, I., Lin, F. Y., Vanommeslaeghe, K., Lemkul, J. A., Armacost, K. A., Brooks, C. L., 3rd, \& Mackerell, A. D., Jr (2016).
Parametrization of halogen bonds in the CHARMM general force field: Improved treatment of ligand-protein interactions. Bioorganic \& medicinal chemistry, 24 (20), 4812-4825. https://doi.org/10.1016/j.bmc.2016.06.034 - Weaver, S. C., Osorio, J. E., Livengood, J. A., Chen, R., \& Stinchcomb, D. T. (2012). Chikungunya virus and prospects for a vaccine. Expert Review of Vaccines, 11 (9), 1087-1 101. doi:10.1586/erv.12.84

- Ziegler, S. A., Lu, L., da Rosa, A. P., Xiao, S. Y., \& Tesh, R. B. (2008). An animal model for studying the pathogenesis of chikungunya virus infection. The American journal of tropical medicine and hygiene, 79(1), 133-139.

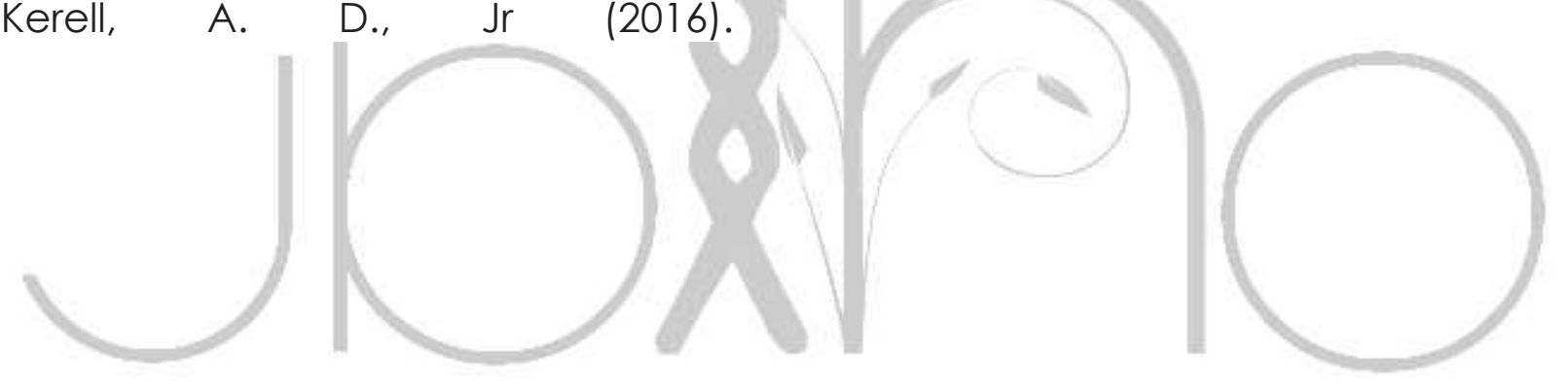

